\title{
LA TERMINACIÓN CONVENCIONAL EN EL DERECHO DE LA COMPETENCIA, ¿UNA SOLUCIÓN ADECUADA A UN PROCEDIMIENTO SANCIONADOR?
}

\author{
Autores: Fernando Díez Estella ${ }^{1}$ \\ Profesor Contratado Doctor de Derecho Mercantil \\ Centro Universitario Villanueva
}

Resumen

El indudable auge de los ADR -mecanismos alternativos para la resolución de conflictos- ha tenido también su reflejo en el ámbito antitrust, ya que uno de los objetivos declarados de las reformas acometidas por la Ley 15/2007, de Defensa de la Competencia, fue la flexibilización del régimen de terminación convencional -los ATC- como mecanismo de finalización de los expedientes sancionadores de conductas restrictivas de la competencia. Además de un somero repaso de las cuestiones procedimentales más relevantes, una comparativa entre los modelos en EE. UU., la UE y España, y el análisis del tipo de compromisos que suelen

\footnotetext{
${ }^{1}$ fdiez@villanueva.edu
} 
ofrecerse, el objetivo de este trabajo es señalar cuáles son los problemas más habituales que se suscitan en la práctica, indagando en los motivos por los que los ATC no han tenido el "éxito" que se esperaba de ellos.

Palabras clave: terminación convencional; expediente sancionador; compromisos; multas.

\section{Settlements in antitrust law: an adequate solution for sanctioning proceedings?}

\section{Abstract}

The recent increase in using the so-called ADR -alternative dispute resolutionhas also reached antitrust law. In Spain, one of the declared aims of Competition Defence Act 15/2007 was encouraging this sort of termination of sanctioning proceedings. However, so far the results obtained are clearly under the expectations placed. We examine the reasons of this 'failure', taking also into account the relevance of the necessarily joined tool to reach such an agreement: the commitments offered by the undertaking suspected of infringing antitrust law. We show the differences between US and EU framework, when compared with the Spanish scheme of settlements.

Key words: settlements; commitments; antitrust proceedings; sanctions.

\section{INTRODUCCIÓN}

En el marco -más amplio- de la búsqueda de mecanismos alternativos para la resolución de conflictos (en inglés, ADR alternative dispute resolution), no cabe duda que uno de los objetivos declarados de las reformas acometidas por la Ley 15/2007, de Defensa de la Competencia, fue la flexibilización del régimen de terminación convencional como mecanismo de finalización de los expedientes sancionadores de conductas restrictivas de la competencia.

Para contextualizar el tema que va a ser objeto de análisis en estas páginas, sirvan unas acertadas palabras de la doctrina administrativista:

"El acuerdo entre el interesado y la Administración que bien tiene la consideración de acto terminal del procedimiento administrativo dentro del 
cual se adopta, o bien fija con carácter vinculante los datos que han de ser tenidos en cuenta al dictar el acto terminal correspondiente, o bien sirve de antecedente no vinculante de dicho acto terminal"

En efecto, hablar de terminación convencional significa, en primer lugar, hablar de un acuerdo entre el interesado y la Administración, y sólo esta consideración previa sitúa en su justo contexto el resto de reflexiones que se puedan hacer después.

En nuestro país esta figura está contemplada, en el ámbito del Derecho Administrativo, por el Art. 88 de la Ley 30/1992, de 26 de noviembre, de Régimen Jurídico de las Administraciones Públicas y del Procedimiento Administrativo Común, y en el ámbito específico del Derecho de la Competencia, por el Art. 52 de la Ley 15/2007, de Defensa de la Competencia ${ }^{3}$ (en adelante, LDC), y el Art. 39 del R. D. 261/2008, de 22 de febrero, por el que se aprueba el Reglamento de Defensa de la Competencia ${ }^{4}$ (en adelante, RDC).

Así mismo, y desde la perspectiva del Derecho comunitario, la normativa aplicable es el artículo 7 del Reglamento (CE) n ${ }^{\circ}$ 1/2003 del Consejo, de 16 de diciembre de 2002, relativo a la aplicación de las normas sobre competencia previstas en los artículos 81 y 82 del Tratado ${ }^{5}$, así como el Reglamento (CE) n ${ }^{\circ}$ 622/2008, de la Comisión, de 30 de junio, por el que se modifica el Reglamento (CE) n ${ }^{\circ} 773 / 2004$ de transacción en casos de cártel ${ }^{6}$.

Como es bien sabido, en el ámbito del Derecho de Defensa de la Competencia es abundante y profusa la existencia de documentos de soft law, que sin tener un valor legal directamente vinculante, sirven de guía a las autoridades antitrust y de orientación a los administrados sobre la aplicación de la normativa en esta materia.

Por ello, necesariamente hemos de referirnos aquí, para completar este "cuadro normativo" en materia de terminación convencional, a la Comunicación de la Comisión sobre el desarrollo de los procedimientos de transacción con vistas a la adopción de decisiones con arreglo a los arts. 7 y 23 del Reglamento (CE) núm. 1/2003 del Consejo en casos de cártel ${ }^{7}$-en el ámbito comunitario-, y la Comunicación sobre terminación convencional de Expedientes Sancionadores de la Comisión Nacional de Competencia ${ }^{8}$, en nuestro derecho interno.

${ }^{2}$ González Pérez, J., Comentarios a la Ley de Régimen Jurídico de las AA. PP. y PAC, Madrid, 2004, p. 2167.

${ }^{3}$ Ley 15/2007, de 3 de julio, de Defensa de la Competencia, BOE núm. 159, de 4 de julio de 2007, p. 12496.

${ }^{4}$ Real Decreto 261/2008, de 22 de febrero, por el que se aprueba el Reglamento de Defensa de la Competencia, BOE núm. 50, de 27 de febrero de 2008, pp. 11575-11604.

${ }^{5}$ DOCE L 1, de 4 de enero de 2003, pp. 1-15.

${ }^{6}$ DOCE L 171, de 1 de julio de 2008, pp. 3-6.

${ }^{7}$ DOCE C 167, de 2 de julio de 2008, pp. 1-6.

${ }^{8}$ Disponible en la web: http://www.cncompetencia.es/Inicio/GestionDocumental/tabid/76/Default.as px?Entryld=104314\&Command=Core_Download\&Method=attachment 
No se trata en este artículo de detallar los procedimientos y requisitos propios de esta peculiar forma de acabar un procedimiento sancionador en el ámbito del Derecho de la Competencia, los acuerdos de terminación convencional (en adelante ATC), para ello nos remitimos a los trabajos ya publicados ${ }^{9}$, tanto sobre el ordenamiento comunitario como el español. No obstante, y para situar nuestro análisis ulterior, se llevará a cabo, en el epígrafe 2, un somero repaso de las cuestiones procedimentales más relevantes.

El objetivo de este trabajo es señalar cuáles son los problemas más habituales que se suscitan en la práctica, indagando en los motivos por los que, hasta ahora por lo menos, los ATC no han tenido el "éxito" que se esperaba de ellos. A ello se dedica el epígrafe 3. Quizá, parte del problema sea la cuestión que va ineludiblemente unida a la terminación convencional: los compromisos, que analizamos en el epígrafe 4. Finalmente, se ofrece un último apartado 5 de Conclusiones.

\section{EL SISTEMA DE TERMINACIÓN CONVENCIONAL}

En este epígrafe se pretende es señalar, tanto en el ámbito comunitario (2.1) como en el español (2.3) los principales aspectos a tener en cuenta, y que han resultado más problemáticos en cuanto a su implementación práctica. También se lleva a cabo una breve reflexión así como un estudio comparativo entre el modelo europeo y el norteamericano (2.2), destacando las divergencias entre ambos al hilo de los conocidos casos Google y Microsoft. Dedicamos un último apartado (2.4) una primera valoración de las ventajas e inconvenientes del sistema.

Pese a que diversas leyes especiales tienden a facilitar la consecución de acuerdos de la Administración con los particulares, la figura de la TC como forma de finalización de un expediente sancionador no cabe duda que suscita un gran interés tanto teórico como práctico. Se plantea no sólo su incompatibilidad con Artículo 22 del Real Decreto 1398/1993, de 4 de agosto, por el que se aprueba el Reglamento del Procedimiento para el Ejercicio de la Potestad Sancionadora, sino incluso cuestiones de su validez constitucional, que han sido resueltas favorablemente a la TC y de forma unánime - por lo menos en el ámbito de la defensa de la competencia- por la jurisprudencia ${ }^{10}$ y la doctrina ${ }^{11}$. Una vez, por tanto

\footnotetext{
${ }^{9}$ Vid., por todos, Tudor, C., "La transacción y terminación convencional de los procedimientos sancionadores en materia de defensa de la competencia", en Velasco, L. A. (dir), Acuerdos horizontales, mercados electrónicos y otras cuestiones actuales de competencia y distribución, Ed. Lex Nova - Thomsom Reuters, Valladolid, 2014, pp. 278-308.

${ }^{10}$ Sentencia del Tribunal Constitucional, de 26 de abril de 1990 (STC 76/90).

${ }^{11}$ Vid., por todos, Baño León, J. M., Voz "Artículo 52. Terminación convencional”, en Folguera, J.

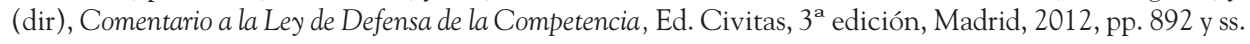


admitida sin vacilaciones su plena validez, intentaremos en las páginas que siguen destacar cuáles son sus rasgos esenciales y los problemas que suscita en la práctica.

\subsection{En la Unión Europea}

El sistema de terminación convencional se introduce en la Unión Europea con el Reglamento 1/2003, en general, y el Reglamento de transacción en casos de cártel, de 2008, constituyendo un elemento clave de la siempre feroz lucha de las autoridades antitrust comunitarias contra los cárteles.

Desde 2010, se han cerrado por acuerdo un total de 17 expedientes sancionadores, ocho de los cuales corresponden al año 2014. En una conferencia ${ }^{12}$ el 8 de marzo de 2013, el anterior Comisario de Competencia "vaticinó" que en un futuro próximo la mitad de los expedientes sancionadores en esta materia se cerrarían a través de un ATC.

Lo cierto es que, echando un vistazo a la evolución de los datos ${ }^{13}$, esta previsión del Comisario Almunia aún puede resultar incluso algo conservadora:

\begin{tabular}{|c|c|c|c|c|c|c|}
\hline Año & $\begin{array}{c}\mathrm{N}^{\text {o de- }} \\
\text { cisiones } \\
\text { de cártel } \\
\text { (total) }\end{array}$ & $\begin{array}{c}\mathrm{N}^{\circ} \text { de } \\
\text { ATC }\end{array}$ & $\begin{array}{c}\% \text { sobre } \\
\text { el total de } \\
\text { decisiones }\end{array}$ & $\begin{array}{c}\text { Total de } \\
\text { multas por } \\
\text { cárteles } \\
(€ \mathrm{M})\end{array}$ & $\begin{array}{c}\text { Total de } \\
\text { multas por } \\
\text { ATC } \\
(€ \mathrm{M})\end{array}$ & $\begin{array}{c}\text { \% sobre el } \\
\text { total de las } \\
\text { multas }\end{array}$ \\
\hline 2010 & 7 & 2 & $28 \%$ & 2,868 & 506 & $18 \%$ \\
\hline 2011 & 4 & 3 & $75 \%$ & 614 & 604 & $98 \%$ \\
\hline 2012 & 5 & 2 & $40 \%$ & 1,876 & 154 & $8 \%$ \\
\hline 2013 & 4 & 2 & $50 \%$ & 1,883 & 1,710 & $91 \%$ \\
\hline 2014 & 10 & 8 & $80 \%$ & 1,689 & 1,250 & $74 \%$ \\
\hline
\end{tabular}

Como ha sido puesto de manifiesto por reputados y prestigiosos miembros de la Unidad de Cárteles en la DG COMP, "una «transacción» no es sino una decisión que constata y penaliza una infracción, adoptada en virtud de los arts. 7 y 23 del Reglamento núm. 1/2003 al cabo de un procedimiento simplificado en el curso del cual los destinatarios de la decisión han admitido su responsabilidad por la infracción del art. 81 CE [hoy es el artículo 101 TFUE]. Por tanto, la transacción

\footnotetext{
12 "Remedies, commitments and settlements in antitrust",SV Kartellrecht, Brussels, (SPEECH/13/210) 8 March 2013, disponible en: http://europa.eu/rapid/press-release_SPEECH-13-210_en.htm

${ }^{13} \mathrm{http}: / /$ ec.europa.eu/competition/cartels/statistics/statistics.pdf
} 
comunitaria no constituye una alternativa informal a un procedimiento formal ordinario. Se trata, por el contrario, de un procedimiento reglado, aunque más ágil, conducente a una decisión formal susceptible de revisión por la judicatura competente" 14 .

Los elementos claves del procedimiento comunitario para un ATC se pueden resumir en los siguientes:

- Total discrecionalidad de la Comisión Europea para iniciar o no el procedimiento.

- Preferencia, por parte de las autoridades, de que todas las partes implicadas participen en el ATC.

- Posibilidad de "salirse" del acuerdo -opt out- en cualquier momento hasta que se envíe la propuesta formal.

- 10\% de descuento en la multa, acumulable al descuento propio de los programas de clemencia.

- Procedimiento sumario, diseñado para una mayor rapidez procesal.

- Tras un contacto inicial, posibilidad de acceso al expediente de la Comisión y evidencia probatoria en la que se va a fundamentar la responsabilidad, gravedad de la sanción, atenuantes, multas, etc.

- Mientras que las discusiones previas no implican autoinculpación, la propuesta formal de acuerdo sí exige "reconocimiento inequívoco de responsabilidad por la infracción".

- El procedimiento es ágil (actualmente está llevando entre 8 y 10 meses), en principio es confidencial, y se respetan mucho los derechos de defensa y posibilidad de recurso (en atención al reconocimiento de responsabilidad).

Es importante advertir ${ }^{15}$ desde un principio que el sistema de ATC que se ha configurado en la UE difiere sensiblemente del modelo en el que se inspiró, el norteamericano ${ }^{16}$, y es también distinto del que tenemos en nuestro país ${ }^{17}$. En

\footnotetext{
${ }^{14}$ Tierno Centella, M ${ }^{a}$ L., "El procedimiento comunitario de transacción para asuntos de cárteles", en Martínez Lage, S. y Amadeo Petitbò, J. (dir), Los Acuerdos Horizontales entre Empresas, Ed. Fundación Rafael del Pino, Vol 28 (2009), p. 372.

${ }^{15}$ Siragusa, M. y Guerri, E., "Antitrust settlements under EC Competition Law: the Point of view of the Defendants", en Ehlermann, C. y Marquis, M. (dirs), European Competition Law Annual 2008, Hart Publishing, Oxford, 2010, pp. 193 y ss.

${ }^{16}$ Vid., para un desarrollo extenso sobre las similitudes y diferencias, O’Brien, A., "Cartel settlements in US and EU: similarities, differences and remaining questions", 13th Annual EU Competition Law and Policy Workshop, Florence, 6 de junio de 2008, págs. 2 y ss. Disponible en la página web del DOJ: http:// justice.gov/atr/public/speeches/235598.pdf

${ }^{17}$ Guzman Zapater, C. y Fornells De Frutos, J., "La terminación convencional en la Ley 15/2007 de Defensa de la Competencia a la luz de la Comunicación de la CNC" (December 3, 2013). Working Paper IE Law School AJ8-197. Disponible en: http://ssrn.com/abstract=2407052
} 
efecto, conforme a la LDC, y como ha señalado la jurisprudencia, y examinaremos en el apartado siguiente "la existencia de infracción es incompatible con un ATC" 18 .

La valoración que se ha hecho, hasta ahora, es positiva ${ }^{19}$; sin dejar de reconocer las carencias del sistema y sus inconvenientes, la práctica totalidad de la doctrina que ha estudiado el procedimiento, y los abogados que han aconsejado a sus clientes acudir a esta vía, están satisfechos con la terminación convencional en la UE.

\subsection{El modelo de consent decree estadounidense}

En EE. UU. el Tribunal Supremo no reconoció la constitucionalidad de los settlement procedure hasta la conocida Sentencia US v. Brady ${ }^{20}$, de 1970. Como se ha señalado, a día de hoy "dos principios generales gobiernan la utilización de la terminación convencional en un procedimiento sancionador en EE. UU. en materia antitrust. Primero, la celebración de un acuerdo en este sentido es considerada un derecho legítimo, aunque no constitucional, de las partes (...). Segundo, si las partes deciden optar por la terminación convencional de un procedimiento, el proceso de negociación debe realizarse respetando determinadas reglas procesales para garantizar que el acuerdo alcanzado sea justo, transparente, libre y equilibrado" 21 .

No se trata aquí de entrar al detalle de esas reglas procesales, nos remitimos para ello a los trabajos publicados por la doctrina ${ }^{22}$. Sin embargo, sí queremos llamar la atención, al hilo de dos recientes -y notorios- casos, los asuntos Microsoft y Google, cómo las divergencias transatlánticas en la aplicación del Derecho antitrust encuentran en este ámbito, el de la terminación convencional, una de sus máximas expresiones.

Esto es así ya que en EE. UU. la terminación convencional es un pilar esencial de su política antitrust, especialmente en la lucha contra los cárteles. En los últimos 20 años se calcula que más del 90\% de los expedientes iniciados por la FTC (Federal Trade Commission) o el DOJ (Departament of Justice, Antitrust Division)

\footnotetext{
${ }^{18}$ Sentencia de la Audiencia Nacional (Sala de lo Contencioso-Administrativo, Sección $6^{\mathrm{a}}$ ), de 26 de marzo de 2013 (JUR 2013 \126127), Fundamento de Derecho n ${ }^{\circ} 7$.

${ }^{19}$ Bellis, J-F., "General Overview of the EU Cartel Settlement Procedure", Competition Policy International, 2014, disponible en: https://www.competitionpolicyinternational.com/assets/General-Overviewof-the-European-Settlement-Procedure.pdf

${ }^{20}$ U. S. v Brady, 397 US 742, $752-753$ (1970).

${ }^{21}$ Tudor, C., op.cit., p. 281.

${ }^{22}$ Reindi, A., "The legal framework governing negotiated settlements / plea agreements in Cartel cases in the United States", en Ehlermann, C. y Marquis, M. (dirs), European Competition Law Annual 2008, Hart Publishing, Oxford, 2010, pp. 62 y ss.
} 


\section{Fernando Díez Estella}

han acabado en un acuerdo, que en su legislación recibe el nombre específico de 'consent decree'.

Se ha señalado ${ }^{23}$ cómo estos datos ponen de manifiesto la profunda confianza del sistema en que tanto las partes interesadas como la administración pública tienen capacidad $-\mathrm{y}$ voluntad política- de llegar a un acuerdo, que reconduzca los principios de eficiencia y mejora del mercado en el caso concreto, una vez producida la infracción.

Sin embargo, y así lo ha puesto de manifiesto la doctrina más atenta ${ }^{24}$, la distancia que separa ahora mismo los procedimientos de terminación convencional en EE. UU. y en la UE es sencillamente inaceptable, desde el punto de vista de la seguridad jurídica, la predictibilidad económica, y desde luego frontalmente opuesta a los intentos de una cierta armonización entre ambos ordenamientos antitrust.

Por ello, y lamentablemente, compañías multinacionales -como Google y Microsoft- se encuentran con que los mismos procedimientos de terminación convencional seguidos a uno y otro lado del Atlántico acaban con resultados totalmente distintos.

Así, mientras que la investigación sobre el caso Microsoft se cerró en EE. UU. con un 'consent decree' en 2001, en la UE terminó con la histórica multa ${ }^{25}$ de 457 millones de euros en 2004, confirmada a los tres años por Sentencia ${ }^{26}$ del entonces TPI (Tribunal de Primera Instancia), hoy Tribunal General.

Por su parte, en el caso Google, en Estados Unidos la investigación se cerró -al concluirse que no había habido ninguna conducta anticompetitiva- mediante un acuerdo ${ }^{27}$ entre la empresa y la FTC (Federal Trade Commission), y en el ámbito comunitario parecía que lo mismo va a ocurrir con la Comisión Europea, pero cuando todo apuntaba a que estábamos a punto de contemplar el acuerdo, (de hecho su texto ya había sido filtrado ${ }^{28}$ por la propia empresa) una serie de problemas técnicos, y oposición política, impidieron concluirlo.

${ }^{23}$ Vid., por todos, Ginsburg, D. y Wright, J., "Antitrust Settlements: the Culture of Consent" (February 27, 2013), George Mason Law Eु Economics Research Paper num. 13-18, disponible en: http:// papers.ssrn.com/sol3/papers.cfm?abstract_id=2225894

${ }^{24}$ González-Magaz, J. y Gordon, W., "From Microsoft to Google - Continued Divergence in Transatlantic Antitrust Settlement", CPI Antitrust Chronicle, March 2013, num. 3, disponible en: https://www. competitionpolicyinternational.com/from-microsoft-to-google-continued-divergence-in-transatlanticantitrust-settlements/

${ }^{25}$ Decisión de la Comisión, de 24 de marzo de 2004, relativa a un procedimiento de aplicación del artículo 82 del Tratado CE (COMP/C-3/37.792 Microsoft).

${ }^{26}$ Sentencia del TPI de 17 septiembre 2007, As. T-201/04, Microsoft/Comisión.

${ }^{27}$ No estrictamente hablando un 'consent decree', pero legalmente de características y alcance muy similares: In the Matter of Google Inc., FTC File num. 121-0120 January núm. 3, 2013, disponible en: http://www.ftc.gov/sites/default/files/documents/cases/2013/01/130103googlemotorolastmtofcomm.pdf

${ }^{28}$ COMMITMENTS IN Case COMP/C-3/39.740 - Foundem and others, de 31 de enero de 2014, texto íntegro disponible en: http://docs.dpaq.de/6448-google_commitments_full.pdf. 
Con las elecciones al Parlamento Europeo y el nombramiento de la nueva Comisaria de Competencia, Margarethe Vestager no sólo no se ha avanzado hacia una terminación convencional del expediente, sino que todo apunta ${ }^{29}$ a que la DG COMP terminará sancionando al buscador norteamericano, ... ¡la historia se repite!

\subsection{La configuración en España de los ATC}

Hemos señalado en la introducción que la base legal en nuestro país para llegar a un ATC se encuentra en el artículo 52.1 LDC. Conforme a sus previsiones, para que proceda la terminación convencional de un expediente deben cumplirse -de forma cumulativa, como ya ha recogido la jurisprudencia ${ }^{30}-$ los siguientes requisitos:

1. Tiene que haberse incoado un expediente sancionador por parte del órgano administrativo correspondiente (que puede ser, naturalmente, la actual CNMC, o bien alguna de las autoridades autonómicas de defensa de la competencia).

2. Dicho expediente sancionador ha de haberse incoado en materia de conductas y prácticas restrictivas prohibidas por la LDC (es decir, las contempladas $^{31}$ en los artículos 1,2 y 3 , y quedando por tanto excluidas de este procedimiento las concentraciones empresariales ${ }^{32}$ ).

3. En cuanto al marco temporal, la terminación convencional puede iniciarse en cualquier momento, pero siempre antes de haberse producido el Informe Propuesta por parte de la DI (Dirección de Investigación).

4. Finalmente, junto al ATC tiene que haber una propuesta de compromisos por parte de los presuntos infractores.

A este respecto, la Comunicación de la CNC sobre terminación convencional ${ }^{33}$ de 2011 recuerda, por si quedaba alguna duda, "que la terminación convencio-

${ }^{29}$ Statement by Commissioner Vestager on Google antitrust investigations at the European Parliament (ECON committee meeting), Brussels, 11 November 2014, disponible en: http://europa.eu/rapid/ press-release_STATEMENT-14-1646_en.htm

${ }^{30}$ Sentencia de la Audiencia Nacional (Sala de lo Contencioso-Administrativo, Sección $6^{\text {a }}$ ), de 9 de mayo de 2013 (JUR 2013\161776), Fundamento de Derecho, num. 2.

${ }^{31}$ Velasco San Pedro, L., "La terminación convencional del procedimiento sancionador en materia de defensa de la competencia", en Revista de Derecho de la Competencia y la Distribución, n 4, 2009, pp. 174 y ss.

${ }^{32}$ En todo caso, el párrafo n 7 de la Comunicación de 2011 no deja lugar a dudas: "La resolución de un expediente sancionador mediante terminación convencional constituye una forma de finalizar un procedimiento sancionador incoado por una posible infracción sustantiva de la legislación de defensa de la competencia, es decir, por infracción de los artículos 1, 2 y/o 3 LDC".

${ }^{33}$ COMUNICACIÓN SOBRE TERMINACIÓN CONVENCIONAL DE EXPEDIENTES SANCIONADORES, disponible en: http://www.cncompetencia.es/Inicio/GestionDocumental/tabid/76/Default.aspx?EntryId=104314\&Command=Core_Download\&Method=attachment 


\section{Fernando Díez Estella}

nal es una forma de finalización del expediente sancionador distinta del acuerdo transaccional de la normativa comunitaria, que no encuentra reflejo en la regulación española de competencia" (párrafo $n^{\circ} 15$ ). Por su parte, la jurisprudencia ha precisado que se trata de una facultad discrecional de la autoridad de competencia, en ningún caso es un "derecho subjetivo de las empresas" ${ }^{34}$. Esto, naturalmente, ha provocado los correspondientes recursos, habitualmente si se negaba esta vía de terminación del expediente, por entender que generaba "indefensión" 35 o que la autoridad de competencia había interpretado de forma "arbitraria" 36 el cumplimiento de los correspondientes compromisos.

Como ya ha reiterado la incipiente jurisprudencia que empieza a ocuparse de los ATC o la vigilancia / cumplimiento de los compromisos, la discrecionalidad de que goza la Administración no significa -como han intentado en vano alegar algunas empresas en relación a procedimientos de terminación convencional"incongruencia omisiva y ausencia de motivación" 37.

El objeto de los ATC es doble, y lo señala de forma concisa el párrafo ${ }^{\circ} 10$ de la Comunicación de 2011.

Por un lado, se busca lograr un restablecimiento rápido de las condiciones de competencia que se han puesto en riesgo con las conductas restrictivas detectadas, mediante unos compromisos que resuelvan los problemas de competencia o eliminen las restricciones de competencia injustificadas, salvaguardando el bienestar de los consumidores y el interés público.

Por otro lado, permite cumplir con el principio de eficacia administrativa, posibilitando una utilización más adecuada de los recursos de la CNMC, al facilitar una reducción de los trámites de instrucción y un acortamiento de los plazos de resolución del expediente sancionador en el que se acuerda la terminación convencional.

Indudablemente, un especial hito en la configuración de este instrumento en nuestro país fue la publicación, por la entonces $\mathrm{CNC}$, de la ya referida Comunicación sobre terminación convencional, en 2011. Con carácter previo se sometió el texto a consulta pública, y hay que decir que no todos los comentarios ${ }^{38}$ fueron elogiosos.

\footnotetext{
${ }^{34}$ Sentencia de la Audiencia Nacional (Sala de lo Contencioso-Administrativo, Sección 6ª), de 9 de mayo de 2013 (JUR 2013\161776), Fundamento de Derecho núm. 3.

${ }^{35}$ Sentencia de la Audiencia Nacional (Sala de lo Contencioso-Administrativo, Sección 6 $6^{\mathrm{a}}$ ), de 5 de julio de 2012 (JUR 2012 \248386).

${ }^{36}$ Sentencia de la Audiencia Nacional (Sala de lo Contencioso-Administrativo, Sección $6^{\text {a }}$ ), de 23 de diciembre de 2013 (JUR 2014\146).

${ }^{37}$ Sentencia de la Audiencia Nacional (Sala de lo Contencioso-Administrativo, Sección $6^{\text {a }}$ ), de 30 de mayo de 2012 (JUR 2012\195559), Fundamento de Derecho núm. 4.

${ }^{38}$ Vid., a modo de ejemplo, los formulados por la AEDC (Asociación Española para la Defensa de la Competencia), COMENTARIOS DE LA ASOCIACIÓN ESPAÑOLA DE DEFENSA DE LA COMPETENCIA AL PROYECTO DE COMUNICACIÓN SOBRE TERMINACIÓN CONVENCIONAL, Madrid, 15 de julio de 2011, disponible en la web: http://aedc.es
} 
La opinión más generalizada en la doctrina sobre el modelo español es la de poner de relieve $\mathrm{e}^{39}$ los avances que la Ley 15/2007 introduce respecto del sistema anterior, en el sentido de introducir mayor claridad procedimental y transparencia en la negociación tanto entre las partes como frente a terceros, alertando a la vez sobre cuestiones que quedan todavía sin resolver.

\subsection{Valoración preliminar}

A estas alturas estamos ya en condiciones de hacer una primera valoración de las ventajas e inconvenientes del procedimiento de ATC, especialmente en el ámbito comunitario, ya que el español será objeto de estudio en el siguiente epígrafe.

Quedan, además, algunas cuestiones que están todavía sin resolver, como por ejemplo, las razones ${ }^{40}$ por las que el procedimiento de terminación convencional se reserva para los casos de cárteles, y no se aplica a conductas de abuso de posición dominante -como sí ocurre en España- o si realmente ha supuesto un ahorro sensible de recursos materiales y humanos en el trabajo de la Comisión Europea.

Así, como elementos favorables a los ATC, podemos destacar:

- La reducción del 10\% de la multa, con independencia del programa de clemencia.

- Un acceso temprano al expediente de la Comisión, y la evidencia probatoria en la que se fundamenta la acusación, lo que permite una mejor estrategia de defensa.

- Un procedimiento más abierto y dialogante con la autoridad, con mayores oportunidades para que las partes expongan sus argumentos y justificaciones.

- En relación con lo anterior, si la Comisión quiere llegar a un acuerdo, es mucho más receptiva que en un procedimiento sancionador a los argumentos de defensa de los acusados, con lo que se consigue reducir el importe de la sanción.

- Al no haber un Pliego de Cargos completo y Decisión -en toda su extensión-, se evita la publicación de material que luego puede ser utilizado en acciones de daños.

- Siempre está la opción de "levantarse de la mesa" si las exigencias de la Comisión para llegar al ATC no parecen razonables.

\footnotetext{
${ }^{39}$ Especialmente elocuente en este sentido es la opinión manifestada en Araujo Boyd, M., "La Terminación Convencional de los procedimientos en la nueva Ley de Defensa de la Competencia", en Creus, A., (coord.) La Ley 15/2007, de Defensa de la Competencia, Ed. La Ley, Monografía n 1/2008, Madrid, 2008, pp. 435 y ss.

${ }^{40}$ Vid., a este respecto, Tierno Centella, $\mathrm{M}^{\mathrm{a}}$ L., op.cit., p. 375.
} 


\section{Fernando Díez Estella}

- Lo ágil del proceso y la rapidez de resolución ahorra importantes costes legales y que la dirección de la empresa esté "distraída" con el procedimiento antitrust.

Por otro lado, podemos señalar los siguientes aspectos desfavorables:

- El procedimiento de ATC requiere, en última instancia, un "reconocimiento inequívoco de las partes de su responsabilidad por la infracción".

- La decisión suele adoptarse en un plazo de tiempo inferior al de un procedimiento sancionador "normal", lo que implica pago de la multa antes.

- El reconocimiento de responsabilidad -auténtica auto inculpación- puede perjudicar al interesado, bien en procedimientos antitrust que se estén siguiendo en otras jurisdicciones, bien en acciones "follow-on" de daños y perjuicios.

- El acceso al expediente es limitado, aunque en la práctica esto no difiere de cualquier otro procedimiento normal de expediente sancionador.

- El reconocimiento explícito de responsabilidad y la aceptación del Pliego de Cargos (Statement of Objections) limita enormemente las posibilidades de recurso.

Por otro lado, aunque de momento el sistema está funcionando razonablemente bien, y no se está haciendo un uso abusivo de los ATC, es evidente que existe margen para un recurso torticero a los procedimientos de terminación convencional, por ambas partes, tanto por parte de la Comisión Europea (ocultando evidencia exculpatoria, no dando opción a iniciar el procedimiento, aprovecharse de los documentos / evidencia obtenida en las conversaciones y la negociación para usarlos en un procedimiento sancionador normal, castigo a las empresas por no llegar a un acuerdo, malinterpretar sus argumentos, etc.) como de los interesados (negándose a si quiera entablar una negociación preliminar, empezar las conversaciones para ver las "cartas" de la Comisión y luego marcharse, dilación del proceso, infracción de confidencialidad, etc.).

\section{AlgUNOS EJEMPLOS Y PROBLEMAS DE LA PRÁCTICA DE ATC}

Mientras que en los apartados precedentes hemos intentado ofrecer una panorámica general $-\mathrm{y}$ necesariamente somera, por razones de espacio- sobre la terminación convencional en las dos principales jurisdicciones antitrust, la estadounidense y la comunitaria, y explicar en qué medida el modelo español de ATC sigue o se desmarca de ambas, en este apartado nos centraremos en algunos casos y problemas que se han suscitado en la práctica en nuestro país. 
Al hilo de los principales expedientes finalizados por la $\mathrm{CNC}-\mathrm{o}$ la actual CNMC- desde la entrada en vigor de la Ley 15/2007, así como de la valoración que desde diversos sectores se ha hecho de la Comunicación de 2011, pretendemos ilustrar aquí cuál es la realidad de la terminación convencional en España.

Sirva como anticipo de lo que veremos a continuación, que en la gran mayoría de la doctrina ${ }^{41}$ de nuestro país la terminación convencional parece tener ventajas para todo el mundo, y apenas inconvenientes: para la autoridad de competencia supone un ahorro de recursos, sin merma del mantenimiento del interés público; y para las empresas -tanto las infractoras como las posibles perjudicadas por la restricción de la competencia- la rapidez del procedimiento, su menor coste, y posibilidad de intervenir, aporta indudables beneficios.

Con la vigencia de la LDC 16/1989, la potestad para adoptar el ATC era del Servicio de Defensa de la Competencia, sin que mediara intervención alguna del entonces Tribunal de Defensa de la Competencia. Éste, sin embargo, era el competente para resolver, previo informe de vigilancia, sobre el incumplimiento de dicho acuerdo, así como sobre la adopción de la multa coercitiva con el fin de hacer cumplir sus términos (ex Artículo 11.5 de la Ley 16/1989).

Además de otros muchos factores, a la especial dificultad en poner de acuerdo a las partes interesadas (denunciantes y denunciados), se le atribuye el escaso éxito de este instrumento con la ley anterior. De hecho, durante los siete años (2000-2007) de vigencia del sistema previsto en la Ley 16/1989, sólo se culminaron tres ATC en ese período ${ }^{42}$.

Los motivos de tan escasa aplicación, que fue calificada por el Libro Blanco para la reforma del sistema español de Defensa de la Competencia como "desalentadora", los achaca el propio texto a esta rigidez del procedimiento, y más concretamente a "la exigencia de que se produzca sólo en primera fase, antes del Pliego de Concreción de Hechos, y deba contar con el acuerdo de todas las partes, incluyendo a los denunciantes" ${ }^{\prime 3}$.

Sobre esta última cuestión hay una cierta polémica doctrinal, en el sentido de que algunos ${ }^{44}$ autores consideran que no es necesario que todos los presuntos infractores estén de acuerdo con la terminación convencional, y basta con que ellos ofrecieran los pertinentes compromisos, pudiéndose continuar el expedien-

${ }^{41}$ Vid., por todos, Creus Carreras, A. y Vázquez De Prada, E., "Terminaciones convencionales", en Baches Opi, S., (coord.), La Ley 15/2007, de Defensa de la Competencia. Balance de su aplicación, Ed. La Ley, Monografía n 4, Madrid, 2010, pp. 286-287.

${ }^{42}$ Resoluciones de 16 de noviembre de 2006, Exp. 2457/02 SISTEMAS DE PAGO, de 15 septiembre 2005, Exp. 2458/03 ASEMPRE/CORREOS, y de de 27 de noviembre de 2003, Exp. 2398/02 DAMA/ SGAE.

${ }^{43}$ Libro Blanco para la reforma del sistema español de Defensa de la Competencia, Publicaciones del Ministerio de Economía y Hacienda, Madrid 20 de enero de 2005, disponible en: http://www.cncompetencia.es/tabid/76/Default.aspx?Entryld=11366\&Command=Core_Download\&Method=attachment

${ }^{44}$ Baño León, J. M., op.cit., p. 624. 


\section{Fernando Díez Estella}

te sancionador con los no firmantes del ATC, mientras que otro ${ }^{45}$ sector doctrinal no admite esta posibilidad. La jurisprudencia, desde luego, abona ${ }^{46}$ la primera de las tesis.

Con los cambios introducidos por la Ley 15/2007, acertadamente calificados como "flexibilizadores" 47 del procedimiento de terminación convencional, se ha fomentado un mayor uso de este procedimiento, cosechándose un cierto éxito en el sentido de que durante los primeros cinco años de vigencia de la Ley 15/2007 el Consejo de la CNC resolvió veinte expedientes ${ }^{48}$ sancionadores mediante terminación convencional.

La litigiosidad a la que estos ATC han dado lugar, en sede de jurisdicción contencioso-administrativa, es abundante, y da cuenta de lo difícil que es poder "contentar" a todas las partes implicadas. Así, por ejemplo, cuando la CNC acordaba no iniciar las negociaciones, se ha alegado por parte de una empresa que ello provocaba un "perjuicio irreparable a sus derechos e intereses legítimos"49.

Lamentablemente, y pese a que la transparencia y la mayor accesibilidad a la información a través de la web fue una de las "promesas" que se hicieron cuando se tramitó la creación de la nueva CNMC, en la actualidad carecemos de datos fiables para saber cuántos y cuáles se han tramitado y resuelto desde octubre de 2013. La única vez que figura en la que el nuevo órgano ha finalizado el expediente sancionador por terminación convencional es la Resolución Orona / Excelsior ${ }^{50}$.

Es de destacar que, además de los dos requisitos habituales para la aceptación de los compromisos -que serán examinados en el epígrafe siguiente-- en este pronunciamiento de la CNMC se invoca también la "protección de los consumidores", en tanto que beneficiarios de la terminación convencional.

Lo que sí es posible encontrar son Resoluciones que resuelven recursos contra acuerdos del Director de Competencia que deniegan el inicio de la terminación convencional, como por ejemplo la Resolución Fabricantes de Automóvil ${ }^{51}$.

En los últimos meses de vida de la CNC, previos a su transformación en el nuevo regulador, se resolvieron por terminación convencional dos expedientes

${ }^{45}$ Irisarry, B., "Artículo 52. Terminación convencional”, en Odriozola, M. (dir) / Irisarry, B. (coord.), Derecho Español de la Competencia, Tomo II, Ed. Bosch, Madrid, 2008, pp. 975 y ss.

${ }^{46}$ Sentencia de la Audiencia Nacional (Sala de lo Contencioso-Administrativo, Sección $6^{\text {a }}$ ), de 9 de mayo de 2013 (JUR 2013\161776).

${ }^{47}$ Guzmán Zapater, C. y Forniells De Frutos, J., op.cit., pp. 10 y ss.

${ }^{48}$ Vid., ANEXO I.

${ }^{49}$ Vid., por todas, la Sentencia de la Audiencia Nacional (Sala de lo Contencioso-Administrativo, Sección $6^{\text {a }}$ ), de 31 de octubre de 2013 (JUR 2013 \351898), que expresamente señala (Fto. Jco. $3^{\circ}$ ) "no es necesario que los compromisos sean aceptados por todos los presuntos infractores".

${ }^{50}$ Resolución de la CNMC, de 4 de diciembre de 2014 (Expediente S/DC/502/14).

${ }^{51}$ Resolución de la CNMC, de 11 de septiembre de 2014 (Expediente R/AJ/0271/14). 
que sí merecen ser mencionados aquí: la Resolución CO2-Cervezas ${ }^{52}$, y la Resolución ASM Eु Toreros ${ }^{53}$.

En el primero de ellos, la Dirección de Investigación (DI) incoó expediente sancionador contra una serie de empresas por supuestas conductas prohibidas por el artículo 1 LDC consistentes en un posible acuerdo de fijación de precios, reparto de mercado e intercambio de información.

En su Resolución el Consejo de la CNC acordó, al amparo del artículo 52 LDC, la Terminación Convencional de dicho procedimiento sancionador, sujeta a los compromisos propuestos por las empresas encausadas. En opinión del Consejo, los compromisos presentados por estas empresas eran idóneos y adecuados para resolver los problemas de competencia planteados.

En el segundo de ellos, se abrió expediente sancionador contra la empresa ALL SPORTS MEDIA 66, S. L. y contra diez toreros por una conducta anticompetitiva, consistente en la firma de diversos contratos entre ASM y los toreros, para la gestión conjunta por ASM de los derechos de retransmisión televisiva de las corridas de toros en las que participen estos toreros, contratos que restringían la competencia en España de cara a la celebración de corridas de toros y a su retransmisión televisiva. El expediente tuvo su origen en la denuncia presentada por Asociación Nacional de Organizadores de Espectáculos Taurinos.

Los contratos de los toreros con ASM preveían que esta entidad hiciera una comercialización conjunta de sus derechos de imagen en las principales ferias de España. Lo recaudado de forma conjunta se repartiría entre ASM y los toreros, pudiendo estos últimos llegar a cobrar sin haber toreado en una determinada feria. El empresario que quisiera contratar a cualquiera de los toreros representados por ASM, debería negociar y pagar en bloque por todos los representados.

\section{LOS COMPROMISOS}

El párrafo n ${ }^{\circ} 12$ de la Comunicación de 2011 no deja lugar a dudas sobre cómo tienen que ser los compromisos que las partes infractoras presentan para llegar a un ATC: "la terminación convencional no será admisible en los casos en que por norma general no existen compromisos viables a los efectos del artículo 52 de la LDC, bien de cara a resolver los efectos sobre la competencia de las conductas investigadas o bien para garantizar suficientemente el interés público".

Por tanto, dos son las condiciones exigibles para la aceptación de los compromisos ofrecidos por la empresa o empresas infractoras:

\footnotetext{
${ }^{52}$ Resolución de la CNC, de 18 de septiembre de 2013 (Expediente S/0337/11).

${ }^{53}$ Resolución de la CNC, de 27 de marzo de 2013 (Expediente S/0418/12).
} 


\section{Fernando Díez Estella}

- Que resuelvan adecuadamente los efectos sobre la competencia derivados de las conductas objeto del expediente.

- Que quede suficientemente garantizado el interés público.

A diferencia del procedimiento de terminación convencional español, que hemos visto que no es un acuerdo transaccional equivalente al del Derecho comunitario, a los compromisos que las partes ofrecen sí se les aplica, además de la normativa española, el Artículo 9 del Reglamento 1/2003, de aplicación de las normas contenidas en los artículos 101 y 102 del TFUE ${ }^{54}$.

Como veremos a continuación, este "elemento fundamental" 55 del ATC es, sin embargo, una de las cuestiones que más litigiosidad ha suscitado en la práctica, y su valoración en cada caso concreto -que ha de hacerse tanto desde el punto de vista subjetivo como objetivo ${ }^{56}$ - una cuestión harto complicada. En un expediente no de terminación convencional sino de Control de Concentraciones, ha señalado a este respecto el Tribunal Supremo que "los compromisos deben interpretarse de forma conjunta, coherente y coordinada" 57 .

En efecto, al tratarse el interés público de un concepto jurídico indeterminado, cuyo contenido y alcance concreto habrá de examinarse en cada caso, atendiendo a las peculiares circunstancias y contexto económico de la práctica restrictiva encausada, resulta cuando menos difícil verificar que su mantenimiento queda debidamente asegurado. Es una labor que compete a la CNMC, y que ejerce con el margen de discrecionalidad característico de este tipo de actuaciones administrativas.

Como ha puesto de manifiesto la mejor doctrina ${ }^{58}$ que se ha ocupado de esta cuestión, lo que en ningún caso debería aceptarse son propuestas de compromisos cuya única finalidad sea la condonación de la sanción a las empresas infractoras. Este tipo de compromisos no garantizarían suficientemente el interés público derivado de un eficaz sistema administrativo sancionador, ya que chocarían frontalmente con sus finalidades de prevención general y especial.

Los conflictos habitualmente se generan cuando, fruto de la labor de vigilancia de los compromisos, la CNMC entiende que se han cumplido, y por medio de Resolución los da por extinguidos. La parte que se consideró en su día perjudicada

\footnotetext{
${ }^{54}$ DOCE L 1/1, de 4 enero 2003, pp. 1-23.

${ }^{55}$ Sentencia de la Audiencia Nacional (Sala de lo Contencioso-Administrativo, Sección 6a), de 21 de mayo de 2012 (JUR 2012 \185482), Fto. Jco núm 5, que añade además que tienen que ser "claros en su tenor literal" y "susceptibles de ser vigilados".

${ }^{56}$ Sentencia de la Audiencia Nacional (Sala de lo Contencioso-Administrativo, Sección 6 ${ }^{\text {) }}$, de 20 de marzo de 2013 (JUR 2013 \292).

${ }^{57}$ Sentencia del Tribunal Supremo (Sala de lo Contencioso-Administrativo), de 15 de diciembre de 2014, Rec. núm. 2038/2012, antecedente de hecho num. 2.

${ }^{58}$ Velasco San Pedro, L., op.cit., pp. 175-176.
} 
por la conducta "infractora" es la que, entonces, puede recurrir esa Resolución, por entender que no se han cumplido ${ }^{59}$, que el cumplimiento es incompleto ${ }^{60}$.

Al hilo del conocido expediente ${ }^{61}$ CORREOS, uno de los primeros casos cerrados a través de terminación convencional, durante la vigencia de la anterior LDC de 1989, la CNC elevó al Consejo un Informe de Vigilancia el 3 de diciembre de 2010 en el que consideraba que se estaba incumpliendo el ATC.

El 25 de febrero de 2011, la Dirección de Investigación acordó la incoación del expediente sancionador contra CORREOS, por incumplimiento del ATC y, por lo tanto, por una infracción muy grave conforme a lo previsto en el artículo 62.4.c) de la LDC, imponiéndosele ${ }^{62}$ una sanción de 4.800.000€. El 3 de marzo de 2011 CORREOS interpuso ante la Audiencia Nacional recurso contenciosoadministrativo contra la resolución del Consejo de la CNC de 15 de febrero de 2011, y en el pronunciamiento ${ }^{63}$ que resolvió el recurso la Audiencia Nacional lleva a cabo una precisa delimitación de qué es y qué no es un ATC. Así, señala (Fundamento de Derecho $4^{\circ}$ ):

"La recurrente considera que la resolución impugnada vulnera el ordenamiento jurídico, con fundamento en la naturaleza jurídica del acuerdo de terminación convencional objeto del mismo. A su juicio es un acuerdo tripartito de naturaleza convencional equivalente a una transacción judicial, sin que la Administración pueda modificar unilateralmente la voluntad de las partes. La Sala no comparte esta apreciación de la naturaleza jurídica del acuerdo de terminación convencional: los preceptos de aplicación han sido reproducidos porque revelan con claridad que si bien la iniciativa corresponde a los administrados, y no cualquiera sino 'a propuesta de los presuntos autores de las conductas prohibidas' la decisión corresponde legalmente única y exclusivamente al Consejo de la CNC".

Otra cuestión controvertida es la relativa a la complementariedad de la terminación convencional con la imposición de sanción. A este respecto hay posturas enfrentadas en la doctrina, ya que la LDC ciertamente no contempla esta posibilidad, aunque es cierto que tampoco la prohíbe, y -como hemos tenido oportuni-

\footnotetext{
${ }^{59}$ Sentencia de la Audiencia Nacional (Sala de lo Contencioso-Administrativo, Sección $6^{\mathrm{a}}$ ), de 18 de marzo de 2014 (JUR 2014\110602).

${ }^{60}$ Sentencia de la Audiencia Nacional (Sala de lo Contencioso-Administrativo, Sección $6^{\text {a }}$ ), de 2 de diciembre de 2011 (JUR 2011 \436009).

${ }^{61}$ Acuerdo de Terminación Convencional, de 15 de septiembre de 2005 (Expte. 2458/03), firmado por la Directora General de Defensa de la Competencia, en su calidad de Directora del SDC, y por los Presidentes de CORREOS y de ASEMPRE (esta última empresa fue la denunciante que dio origen al expediente sancionador).

${ }^{62}$ Resolución de la CNC, de 23 de agosto de 2011 (Expte. SNC/0011/11, CORREOS).

${ }^{63}$ Sentencia de la Audiencia Nacional (Sala de lo Contencioso-Administrativo, Sección $6^{\text {a }}$ ), de 25 de abril de 2013 (JUR 2013\133067).
} 
dad de examinar en un epígrafe precedente- sí está expresamente permitida por el procedimiento comunitario de transacción en casos de cártel.

Desde luego ni la extinta CNC ni la actual CNMC han hecho uso hasta ahora de esta posibilidad, y en cambio quien sí ha compatibilizado un ATC con la imposición de multa ha sido el Tribunal Vasco de Defensa de la Competencia. En la Resolución HIRU ${ }^{64}$ llegó a un acuerdo con la empresa infractora, y aceptó los compromisos ofrecidos, a la vez que la consideró responsable de la adopción de una recomendación de aumento de precios dirigida a sus asociados, incursa en la prohibición del artículo 1 de la Ley 15/2007, de 3 de julio, de Defensa de la Competencia, y fue por tanto sancionada con una multa de $25.000 €$.

Ha sido el mismo TVDC quien, en la Resolución Colegio Oficial de Diseñadores de Interior / Decoradores de Guipúzcoa ${ }^{65}$, ha concluido el expediente sancionador por ATC y la aceptación de compromisos ${ }^{66}$ pero esta vez sin imposición de multa alguna.

En el mismo sentido en fechas más recientes, se ha pronunciado el Consejo de Defensa de la Competencia de Andalucía, en su Resolución COAS y CACOA ${ }^{67}$, en la que cierra por terminación convencional un expediente sancionador incoado por presuntas prácticas restrictivas del Colegio Oficial de Arquitectos de Sevilla, con la aceptación -y vigilancia-- de los compromisos ofrecidos, y sin imposición de multa.

A título puramente anecdótico -y si se nos permite la expresión, un tanto folclórico-, el segundo de dichos compromisos era "la organización de unas Jornadas sobre Competencia y Colegios Profesionales, con las especialidades que presentan los servicios de arquitectura, este Consejo lo valora como esencial para que los profesionales colegiados tengan conocimiento del desarrollo óptimo y eficiente de su actividad en el marco de la libre competencia sin incurrir en actuaciones anticompetitivas" (Fundamento de Derecho $3^{\circ}$ ).

Un compromiso similar fue el aceptado para el ATC que se contiene en la Resolución Colegios Notarial de Andalucía ${ }^{68}$, y se le dio cumplimiento con la orga-

\footnotetext{
${ }^{64}$ Resolución del TVDC, de 20 de mayo de 2009, Expte. 01/2009, disponible en la web del organismo vasco de competencia: http://www.competencia.euskadi.eus/resoluciones/ (consultada por última vez el 11 de mayo de 2015).

${ }^{65}$ Resolución del TVDC, de 9 de mayo de 2012, Expte. 09/2011, disponible en la web del organismo vasco de competencia: http://www.competencia.euskadi.eus/resoluciones/ (consultada por última vez el 18 de junio de 2015).

${ }^{66}$ Se incluyen en el ANEXO II.

${ }^{67}$ Resolución del Consejo de Defensa de la Competencia de Andalucía, de 12 de marzo de 2014, Expte. S/09/2014, disponible en la web del organismo andaluz de competencia: http://www.juntadeandalucia. es/defensacompetencia/competencia/Resoluciones/ (consultada por última vez el 21 de junio de 2015).

${ }^{68}$ Resolución del Consejo de Defensa de la Competencia de Andalucía, de 29 de septiembre de 2011, Expte. S/09/2011, disponible en la web del organismo andaluz de competencia: http://www.juntadeandalucia.es/defensacompetencia/competencia/Resoluciones/ (consultada por última vez el 11 de mayo de 2015).
} 
nización unas Jornadas, en cuyas actas ${ }^{69}$ pueden leerse interesantes consideraciones, como la calificación de este peculiar procedimiento de terminación convencional de un camino hacia una "Administración concertada", y diferenciar -con encomiable precisión jurídica- que "terminación convencional y transacción no son una misma cosa".

Señalar, finalmente, que las resoluciones de los órganos autonómicos se recurren ante los respectivos Tribunales Superiores de Justicia. El pretendido incumplimiento parcial de los compromisos impuestos en la Resolución Col-legi D'Economistes de Catalunya ${ }^{70}$ fue recurrido, y en la Sentencia que lo resuelve, se hace una precisión interesante: "los compromisos recogidos en la resolución que ponga término al procedimiento [de terminación convencional] han de ser claros, concretos y concluyentes, de manera que no ofrezcan dudas sobre el alcance y contenido de los mismos" $"$.

\section{CONCLUSIONES}

Para finalizar este análisis de la terminación convencional en el Derecho de Defensa de la Competencia podemos señalar las siguientes cuestiones:

En primer lugar, es evidente que la terminación convencional supone, cuando menos, una forma "original" y atípica de finalización de un expediente sancionador, de ahí que tampoco puede extrañar las dudas que la figura ha suscitado, y la cautela con la que debe afrontarse su empleo, tanto por parte de la Administración como las empresas. Las ventajas que ofrece para ambos, respecto a un procedimiento normal, están también fuera de toda discusión.

En segundo lugar, tras unos comienzos que hemos calificado como "desalentadores", las reformas en los ATC introducidas por la Ley 15/2007, tendentes todas ellas a una mayor flexibilización del procedimiento, han cosechado un éxito indudable en cuanto al número de procedimientos cerrados por esta vía.

En tercer lugar, sorprende un poco que el modelo español no esté alineado con el comunitario, y que haya todavía aspectos tan importantes como la posible transacción de la multa, o la hipotética terminación convencional añadida a la sanción, que siguen sin resolverse.

\footnotetext{
${ }^{69}$ Disponibles en la web: http://notin.es/administracion-concertada/ (consultada por última vez el 11 de junio de 2015).

${ }^{70}$ Resolución de la Autoritat Catalana de la Competència, de 29 de julio de 2010, Expte. 12/2009, que se encuentra disponible en la página web del organismo catalán de defensa de la competencia, http:// www.acco.gencat.cat/web/.content/resol_exp_i_1_2011_cec_esp.pdf (consultada por última vez el 11 de junio de 2015).

${ }^{71}$ Sentencia del TSJ de Cataluña (Sala de lo Contencioso-Administrativo, Sección $5^{\mathrm{a}}$ ), de 5 de junio de 2014, núm. 512/2014 (JUR \2014\237566), Fundamento de Derecho $3^{\circ}$.
} 


\section{Fernando Díez Estella}

En cuarto lugar, es llamativa la litigiosidad que han provocado las Resoluciones de vigilancia de los compromisos, y la multitud de recursos por partes afectadas por decisiones de darlos por extinguidos.

En quinto y último lugar, es de esperar que con el tiempo, y la praxis de las autoridades de competencia así como la delimitación jurisprudencial de los ATC y los compromisos, se llegue a un mejor y más profundo entendimiento y uso de los mismos.

\section{BIBLIOGRAFÍA}

ALMUNIA, J., "Remedies, commitments and settlements in antitrust", SV Kartellrecht, Brussels, (SPEECH/13/210) 8 March 2013, disponible en: http://europa.eu/rapid/press-release_SPEECH-13-210_en.htm

ARAUJO BOYD, M., "La Terminación Convencional de los procedimientos en la nueva Ley de Defensa de la Competencia", en CREUS, A., (coord.) La Ley 15/2007, de Defensa de la Competencia, Ed. La Ley, Monografía $n^{\circ} 1 / 2008$, Madrid, 2008, pp. 435 y ss.

BAÑO LEÓN, J. M., Voz "Artículo 52. Terminación convencional”, en FOLGUERA, J. (dir), Comentario a la Ley de Defensa de la Competencia, Ed. Civitas, $4^{a}$ edición, Madrid, 2015, pp. 1119 y ss.

BELLIS, J-F., "General Overview of the EU Cartel Settlement Procedure", Competition Policy International, 2014, disponible en: https://www.competitionpolicyinternational.com/assets/General-Overview-of-the-European-Settlement-Procedure.pdf

CREUS CARRERAS, A. y VÁZQUEZ DE PRADA, E., "Terminaciones convencionales", en BACHES OPI, S., (coord..) La Ley 15/2007, de Defensa de la Competencia. Balance de su aplicación, Ed. La Ley, Monografía num. 4, Madrid, 2010, pp. 286-287.

GINSBURG, D. y WRIGHT, J., "Antitrust Settlements: the Culture of Consent" (February 27, 2013), George Mason Law $\mathcal{E}$ Economics Research Paper num. 1318, disponible en: http://papers.ssrn.com/sol3/papers.cfm?abstract_id=2225894

GONZÁLEZ PÉREZ, J., Comentarios a la Ley de Régimen Jurídico de las AA. PP. y PAC, Ed. Thomsom-Civitas, Madrid, 2004, p. 2167.

GONZÁLEZ-MAGAZ, J. y GORDON, W., "From Microsoft to Google Continued Divergence in Transatlantic Antitrust Settlement", CPI Antitrust Chronicle, March 2013, num. 3, disponible en: https://www. competitionpolicyinternational.com/from-microsoft-to-google-continued-divergence-in-transatlantic-antitrust-settlements/

GUZMAN ZAPATER, C. y FORNELLS DE FRUTOS, J., "La terminación convencional en la Ley 15/2007 de Defensa de la Competencia a la luz de 
la Comunicación de la CNC" (December 3, 2013). Working Paper IE Law School AJ8-197. Disponible en: http://ssrn.com/abstract=2407052

IRISARRY, B., "Artículo 52. Terminación convencional", en ODRIOZOLA, M. (dir) / IRISARRY, B. (coord.), Derecho Español de la Competencia, Tomo II, Ed. Bosch, Madrid, 2008, pp. 975 y ss.

O'BRIEN, A., "Cartel settlements in US and EU: similarities, differences and remaining questions", 13th Annual EU Competition Law and Policy Workshop, Florence, 6 de junio de 2008, pp.2 y ss. Disponible en la página web del DOJ: http://justice.gov/atr/public/speeches/235598.pdf

REINDI, A., "The legal framework governing negotiated settlements / plea agreements in Cartel cases in the United States", en EHLERMANN, C., y MARQUIS, M., (dirs), European Competition Law Annual 2008, Hart Publishing, Oxford, 2010, pp. 62 y ss.

SIRAGUSA, M. y GUERRI, E., "Antitrust settlements under EC Competition Law: the Point of view of the Defendants", en EHLERMANN, C., y MARQUIS, M., (dirs), European Competition Law Annual 2008, Hart Publishing, Oxford, 2010, pp. 193 y ss.

TIERNO CENTELLA, M ${ }^{a}$ L., "El procedimiento comunitario de transacción para asuntos de cárteles", en SANTIAGO MARTÍNEZ LAGE y AMADEO PETITBÒ JUAN (dir), Los Acuerdos Horizontales entre Empresas, Ed. Fundación Rafael del Pino, Vol 28 (2009), p. 372.

TUDOR, C.: "La transacción y terminación convencional de los procedimientos sancionadores en materia de defensa de la competencia", en VELASCO, L. A. (dir), Acuerdos horizontales, mercados electrónicos y otras cuestiones actuales de competencia y distribución, Ed. Lex Nova - Thomsom Reuters, Valladolid, 2014, pp. 278-308.

VELASCO SAN PEDRO, L.: "La terminación convencional del procedimiento sancionador en materia de defensa de la competencia", en Revista de Derecho de la Competencia y la Distribución, num. 4, 2009, pp. 174 y ss. 
Fernando Díez Estella

ANEXO I. EXPEDIENTES RESUELTOS POR ATC (2007-2013)

\begin{tabular}{|c|c|c|}
\hline $\mathrm{N}^{\circ}$ Expte & Nombre & Fecha \\
\hline S/0127/09 & $\begin{array}{l}\text { CONSEJO GENERAL PROCURADORES } \\
\text { TRIBUNALES }\end{array}$ & 20 de mayo de 2010 \\
\hline S/0156/09 & AISGE & 23 de marzo de 2011 \\
\hline S/0162/09 & SEMILLAS DE GIRASOL & 13 de septiembre de 2010 \\
\hline S/0189/09 & $\begin{array}{l}\text { CONSEJO GENERAL DE LA ARQUI- } \\
\text { TECTURA TECNICA DE ESPAÑA }\end{array}$ & 28 de julio de 2010 \\
\hline S/0203/09 & $\begin{array}{l}\text { COLEGIO AGENTES PROPIEDAD IN- } \\
\text { DUSTRIAL }\end{array}$ & 22 de diciembre de 2010 \\
\hline S/0215/10 & TRANSCALIT & 20 de febrero de 2013 \\
\hline S/0235/10 & $\begin{array}{l}\text { CONVENIOS FEMP-CONSEJOS GENE- } \\
\text { RALES DE COLEGIOS PROFESIONALES }\end{array}$ & 28 de diciembre de 2011 \\
\hline S/0246/10 & GRUPO VOCENTO/GRUPO GODO & 30 de junio de 2011 \\
\hline S/0255/10 & PUNTOS SERVICIO EON & 30 de noviembre de 2010 \\
\hline S/0268/10 & PUNTOS SUMINISTRO IBERDROLA & 12 de mayo de 2009 \\
\hline S/0270/10 & GRUPO MALLA & 30 de marzo de 2012 \\
\hline S/0302/10 & ORONA/OMEGA & 22 de noviembre de 2012 \\
\hline S/0338/11 & SUBARU & 31 de agosto de 2012 \\
\hline S/0348/11 & $\begin{array}{l}\text { UNION INTERPROFESIONAL DE MA- } \\
\text { DRID }\end{array}$ & 19 de febrero de 2013 \\
\hline S/0352/11 & UNION DE CORRECTORES & 14 de diciembre de 2012 \\
\hline S/0371/11 & $\begin{array}{l}\text { AUTORES LITERARIOS MEDIOS AU- } \\
\text { DIOVISUALES }\end{array}$ & 15 de septiembre de 2011 \\
\hline S/0386/11 & IONMED ESTERILIZACION & 18 de febrero de 2013 \\
\hline $2697 / 06$ & CEPSA & 29 de julio de 2009 \\
\hline $2738 / 06$ & GALP ENERGIA & 6 de abril de 2011 \\
\hline $2800 / 07$ & SIGNUS & 20 de mayo de 2010 \\
\hline
\end{tabular}


LA TERMINACIÓN CONVENCIONAL EN EL DERECHO DE LA COMPETENCIA...

\begin{tabular}{|l|l|r|}
\hline N ${ }^{\circ}$ Expte & \multicolumn{1}{|c|}{ Nombre } & \multicolumn{1}{c|}{ Fecha } \\
\hline S/002/07 & CONSEJO SUPERIOR ARQUITECTOS & 29 de noviembre de 2010 \\
\hline S/0012/07 & PUERTO BARCELONA & 17 de marzo de 2011 \\
\hline S/0020/07 & TRIO+ & 28 de enero de 2010 \\
\hline S/0076/07 & CONVENIO CONTACT CENTER & 13 de septiembre de 2009 \\
\hline S/0077/07 & CONVENIO SEGURIDAD & 17 de marzo de 2009 \\
\hline S/0096/08 & FNMT & 24 de enero de 2011 \\
\hline S/0418/12 & ALL SPORTS MEDIA 66 & 22 de marzo de 2013 \\
\hline S/0337/11 & DISTRIBUIDORES DE CO2 & 20 de septiembre de 2013 \\
\hline
\end{tabular}

\section{ANEXO II. EJEMPLO DE COMPROMISOS EN UN ATC}

\section{PROPUESTA DE COMPROMISOS DEL COLEGIO OFICIAL DE DISEÑADORES DE INTERIOR/DECORADORES DE GIPUZKOA}

1- Como aclaración y ampliación de lo señalado en el art. 10 de los Estatutos del Colegio, se establece expresamente que la Junta de Gobierno, en la tramitación de las solicitudes de colegiación que se le formulen con arreglo a dicho artículo, aceptará como título suficientemente capacitante para el ejercicio profesional en el nivel que le es propio, y, en consecuencia, aprobará la admisión como colegiados de pleno derecho, de los poseedores del título oficial de Técnico Superior de Artes Plásticas y Diseño en Proyectos y dirección de Obras de Decoración establecido por el Real Decreto del Ministerio de Educación y Ciencia de 1 de Septiembre de 1995, nº.1.464/1995, cuyo currículo en el ámbito de la Comunidad Autónoma del País Vasco fue establecido por el Decreto 237/2004, de 30 de Noviembre, del Departamento de Educación, Universidades e Investigación del Gobierno Vasco, todo ello en base a la equivalencia establecida por el R. D.440/1994, de 11.3.94, del Ministerio de Educación. Este compromiso es de aplicación a todas las solicitudes que sean presentadas en la forma establecida estatutariamente, desde el mismo momento en que conste su entrada en las oficinas del Colegio por cualquiera de los medios legalmente admisibles. 
2- Teniendo en cuenta lo establecido en el art.10.1 de la Ley de Colegios Profesionales, en la redacción dada a la misma por la Ley 25/2009, de 22 de Diciembre, se explicitará el criterio anteriormente señalado en la sección de la página web colegial destinada a la información para el acceso a la actividad profesional y su ejercicio, incluyendo el título de Técnico Superior de Artes Plásticas y Diseño en Proyectos y Dirección de Obras de Decoración señalado en el párrafo anterior bajo el epígrafe "Titulaciones oficiales admisibles a efectos de colegiación" dentro del apartado "requisitos para colegiarse" al que se llega seleccionando "solicitud de alta en el Colegio" desde el menú "Ventanilla única" que figura en la pantalla de entrada a la página.

Este compromiso se llevará a cabo en un plazo de quince días naturales desde que se produzca la resolución final del expediente de terminación convencional, sin perjuicio de que la página pueda estar accesible con carácter general en una fecha anterior. 